\title{
Changing time and emotions
}

\author{
Pierre-Yves Geoffard ${ }^{1}$ and Stéphane Luchini ${ }^{2, *}$ \\ ${ }^{1}$ Paris School of Economics, 48 bd Fourdan, 75014 Paris, France \\ ${ }^{2}$ GREQAM-CNRS, Centre de la Vieille Charité, 13236 Marseille Cedex 02, France
}

\begin{abstract}
In this paper, we consider that our experience of time (to come) depends on the emotions we feel when we imagine future pleasant or unpleasant events. A positive emotion such as relief or joy associated with a pleasant event that will happen in the future induces impatience. Impatience, in our context, implies that the experience of time up to the forthcoming event expands. A negative emotion such as grief or frustration associated with an unpleasant event that will happen in the future triggers anxiety. This will give the experience of time contraction. Time, therefore, is not exogeneously given to the individual and emotions, which link together events or situations, are a constitutive ingredient of the experience of time. Our theory can explain experimental evidence that people tend to prefer to perform painful actions earlier than pleasurable ones, contrary to the predictions yielded by the standard exponential discounting framework.
\end{abstract}

Keywords: experience of time; emotions; impatience; anxiety; discount factor; time preference

I come back to the example of the little girl sitting in her bathtub and who dreaded the moment when the nanny would shower her with cold water. She is frightened, but nevertheless, she turns the water-tap herself and trickles some drops of cold water onto her naked arm. [...] As she waited, the little girl's anxiety increased until she could no longer bear it, and hence preferred to trigger, at least partially, the inevitable shock feared in her imagination. But, by this action, she reduced and almost mastered her anxiety, and through this supreme anxiety became a sort of heroine.

Reik, T. (1940). Aus Leiden Freuden. Masochismus und Gesellschaft. (Translated by the authors.)

\section{INTRODUCTION}

The question of how to evaluate and compare future outcomes is obviously a major issue in economics. In this regard, a common assumption, particularly in the canonical model of the exponential discounted utility, first formulated by Samuelson (1937), is that people attribute less weight to experiences that will occur in the future, that is, people discount future outcomes. One common explanation for discounting the future is that people are impatient (see Loewenstein 1992), that is, they prefer pleasures sooner rather than later and, as a consequence, they prefer to experience pleasant situations first and less pleasant or unpleasant ones later. However, over the last decades this model has been widely challenged as experimental evidence has shown that preferences of individuals regularly depart from the exponential discounting predictions (Frederik et al. 2003). In particular, it has been shown that when individuals have to decide on the timing and sequencing of experiences, they are more likely to prefer unpleasant or less pleasant

* Author for correspondence (stephane.luchini@univmed.fr).

One contribution of 12 to a Theme Issue 'Rationality and emotions'. experiences to happen before more pleasant ones (Loewenstein \& Prelec 1991). This suggests a negative time preference, that is, individuals consider that the remote future is more important than the near future. One explanation for such a preference is that the anticipation itself may be a source of pleasure and pain in the present. This would mean that 'we are able to consume events before they occur through anticipation' (Elster \& Loewenstein 1992, p. 224).

In this article, we will present a different explanation of the negative time preference. The basic intuition of our approach is that the experience of time can depend on the emotional valence of events or situations that people anticipate. For instance, when a person anticipates an event that generates a positive emotion, say spending the next holidays on a sunny beach in the Maldives, she may experience impatience and may feel that these longingly awaited holidays will never arrive - that is, the anticipated duration expands. On the other hand, if this same person faces a negative event some time in the future, such as a difficult exam, she may feel anxiety and time seems to fly until this event; it feels to her as if the exam will arrive too quickly. Hence, the sensation is one of time contracting. This 'time experience' is indeed supported by evidence from psychophysiology and neurobiology. Time, therefore, is not exogeneous to the individual, it is elastic, and this will be influenced by the emotion the person experiences. This elasticity of time makes the next holidays seem so far and the exam so close and so salient that if a person could decide on how to sequence these two events, she would rather do her exam first and spend her time on the beach afterwards, which would not be the case for a standard discounted utility maximizer.

The paper is organized as follows. In the first two sections, we present experimental results on time perception and emotions in psychophysiology and neurobiology. In $\S 3$, we present what we mean by 
emotion and define the concept of basic emotion. The fourth section is devoted to the anticipation of future events and how basic emotions interact with intimate time. In $\$ 5$, we explore the behavioural consequences of our theory in the specific case of deciding now how a person should schedule future actions. The final section concludes.

\section{PSYCHOLOGICAL EVIDENCE ON TIME AND EMOTIONS}

Today, there exists a growing literature in experimental psycho-physiology on time perception and emotions. A typical experiment in physiopsychology investigates the effect of emotional stimuli on time perception. By time perception, psychologists usually mean objective experimental measures of subjective time. These measures are, to summarize them briefly, of two types. One is called time perception and asks individuals at the end of an interval how much time they think has elapsed. This is generally captured on an analogue scale. The other, time production, asks subjects to reproduce a previously elapsed time period by pressing a button or saying 'stop' when they think that the same time interval has expired. ${ }^{1}$

To induce emotions in individuals, psychological experiments usually confront them with different pictures representing household objects, spiders and rats, erotic material or bloody human wounds to mention a few. The reactions to these slides are classified by using two main criteria borrowed from the multidimensional analysis of emotions: affective valence and physiological arousal (Lang et al. 1993). ${ }^{2}$ Emotions are thus described in terms of specific characteristics and not in terms of emotional states such as fear, anger or joy (see Elster 1998; Frijda 2000). Affective valence states whether the induced emotion is located in the positive or negative range of pleasure and pain (generally measured through change in face muscle activity in psychophysiology), while arousal refers to the level of physiological activity (generally measured by skin conductance). ${ }^{3}$

Typical time perception experiments are conducted using two different experimental settings. One experiment confronts the subject with a stimulus for a given period of time and measures subjective time perception given specific emotional valence and physiological arousal. Here, experimental results offer some evidence that negative stimuli cause an overestimation of time while positive stimuli cause an underestimation of time (Angrilli et al. 1997). The other experiment measures the time perception of an individual who has to wait for a specific event to happen. It is this experiment that is indeed the one that economists are generally most interested in, because it resembles the typical situation of an economic agent who discounts future events. ${ }^{4}$ However, fewer experiments have been conducted that manipulate the emotional valence in waiting conditions (Langer et al. 1961; Curton \& Lordahl 1974; Edmonds et al. 1981). The problem with waiting time conditions is that subjects are not engaging in any task and one cannot determine if and what people are thinking while they are waiting. It is thus difficult for a psychophysiologist to measure cognitive and emotional activity appropriately (Angrilli et al. 1997). These kinds of experiments found for instance shorter time estimates for subjects in fear of a coming danger than for subjects in neutral condition (Langer et al. 1961). Inversely, Edmonds et al. (1981) show that subjects who are expecting a pleasant experience overestimate the actual time interval (time passes relatively slowly for them). Fraisse (1984) argues that the expectation of an agreeable event 'leads to paying more attention to the passing of time' (p. 24).

Hence, although these experiments on emotions and time differ in their experimental conditions and were not designed with our particular hypothesis in mind, this literature still provides two basic leads: there is a correlation between affective valence, physiological arousal and perceived time and the correlation between valence and perceived time is more likely to be positive for waiting periods.

\section{NEUROBIOLOGY ON TIME AND EMOTIONS}

Neurobiologists also explore the connection between emotions, time perception and decision-making. Recent studies of patients with damage to the orbitofrontal cortex ${ }^{5}$ (OFC) have shown that these patients report experiencing alteration in emotion experience (Hornak et al. 2003; Rolls 2004; Feldman-Barrett et al. 2007). Alteration in emotion experience is usually gathered in these studies through subjective emotion questionnaires (or emotional change questionnaires when addressed to patients after surgery/ brain-injury/illness; Hornak et al. 2003). In these questionnaires, participants report how often they experience specific emotional states in their current daily life such as sadness, anger, fear, happiness and disgust. The study of patients with damage to OFC is thus supposed to provide a good indication of the role of emotions in decision-making, and especially about how they affect economic behaviour, when compared with 'normal' people or to patients with other brain damage outside the OFC (Damasio 1994).

More specifically, OFC patients have been shown to perform poorly on successive gambling tasks or tests compared with those who have 'intact' emotional processes (Damasio 1994). ${ }^{6}$ For instance, in probabilistic reversal tests, subjects are rewarded or punished probabilistically (using artificial money) on a task of visual discrimination learning and reversal test (see Hornak et al. 2004, for details). OFC patients are usually significantly impaired (while other control patients are not) on the reversal task suggesting that they have difficulties in representing reward and punishmentmore precisely in updating the relation between stimuli and rewards (O'Doherty et al. 2001; Rolls 2004). Interestingly for our paper, it has been argued that patients with OFC lesions have difficulty evaluating appropriately immediate rewards against future losses, owing to an inability to be motivated by mental representation of future states (Damasio 1994; Elster 1998). In general, however, neurobiological studies do not explicitly consider the influence of OFC damage on time perception (Manuck et al. 2003). 
One exception is Berlin et al. (2004). In this study, OFC patients, 'normal' people as well as non-OFC patients with lesions outside the $\mathrm{OFC}^{7}$ undertake a series of tasks (probabilistic reversal test, time perception and production, matching familiar figures, spatial working memory task) and answer a subjective emotion questionnaire as well as different questionnaires that are aimed at measuring impulsivity and personality. In particular, participants estimate time intervals ranging from 10 to $90 \mathrm{~s}$ and also provide a long-term estimation at the end of the entire time perception experiment (participants were asked 'How much time do you think has passed from the moment we started the time task until now?'). First, they show the usual alteration in emotion experience of OFC patients in comparison with 'normal' people and non-OFC patients. Second, however, they also find that OFC patients have a different perception of time from 'normal' patients and non-OFC patients. Results reveal that OFC participants estimated that significantly more time had passed than did 'normal' participants, both for small intervals of time and for the entire experiment. Berlin et al. (2004) argue that OFC patients have a 'faster subjective sense of time' (p. 1114), which would explain why OFC patients overestimate time in both cases. This argument relies on the cognitive psychology concept of an 'internal timer' that 'ticks' at different speeds and thus gives rise to different time perceptions. ${ }^{8}$ For instance, Berlin et al. (2004) argue that OFC patients may have a 'faster cognitive pace (their internal clocks may run faster)' (p. 1120). On the contrary, nonOFC participants report no significant differences from 'normal' people on subjective emotions and present no difference in time perception. ${ }^{9}$ In summary, therefore, from these above-cited neurobiological studies, it can be concluded that there is a correlation not only between emotions, as defined in subjective emotion questionnaires, and time perceptions, but also between emotions, time perception and (in)sensitivity to rewards and punishments.

We will use the results of these studies as ingredients for our analysis of time perception (or, as we will call it, of 'intimate time'). Our approach does not, however, strictly adhere to cognitive studies. Instead of considering an 'internal timer' that gives rise to differences in time perception, we adopt the idea that individuals 'experience' time. As we will explain in $\$ 5$, experience of time will be endogenously induced by a particular sequence of actions or events, which are associated with different emotions. Time is therefore not exogeneously given to the individual by his or her internal clock (even if this clock may tick differently for different people, it is still a clock). In what we are going to present, emotions will therefore be a constitutive ingredient of time experience. ${ }^{10}$ We discuss and define in the next section what we mean by emotions.

\section{TIMELESS BASIC EMOTIONS}

As we have seen in the previous section, there are usually two ways to study emotions in psychology. Descriptions of emotions can be based on common features (emotional valence, arousal, action tendency, etc.) or categorize specific emotional states such as fear, anger, etc. (Elster 1998; Frijda 2000). What seems to be lacking in these two psychological approaches is a definition of what an emotion is that would be instrumental enough to be used as a concept in economic theory. The philosopher Pierre Livet however provides us with a more general definition of emotion (Livet 2002). In his view, an emotion is an affective resonance (physiological and behavioural) induced by a differential between the actual situation and a given (perceived or imagined) situation or event, evaluated according to our actual preferences and affective dispositions. ${ }^{11}$ The larger the differential, the stronger is the emotion. Note that when emotions are represented as a differential, 'surprise' is not a necessary condition for emotions to arise. Hence, we do not rely on any form of 'uncertainty' about future events in the present exercise. We simply assume that emotions are induced by imagining some situation that is different from the actual one. For instance, imagining an oily, greasy meal is enough to induce the emotional experience of disgust-whether or not we may have to eat such a meal in the future. Our analysis shares some elements with the approach of Gilbert et al. (2002). The authors consider that people predict their hedonic reactions to events by first imagining events without including the temporal information (what they call an atemporal representation) to form hedonic reactions to those mental images. ${ }^{12}$

Let us proceed more formally and consider an actual situation that can be located on the scale of pleasure and pain, say at a utility level $u$, and another perceived or imagined situation from which the agent derives utility level $u^{\prime}$. A basic emotion is then defined as the change in utilities from level $u$ to level $u^{\prime}$ and will be noted $e\left(u, u^{\prime}\right)$. Obviously, when there is no change in utilities, no emotion is generated since there is no differential (in utilities) between the current situation and the imagined situation, $e(u, u)=0$. Two other properties of basic emotions can also be derived directly from Livet's idea that emotions increase with the differential. First, when my evaluation $u$ of the current situation increases, while the imagined situation remains the same, the intensity of the emotion decreases. On the other hand, when the evaluation of the future situation $u^{\prime}$ increases and my current situation remains constant, then the emotion increases.

According to these primary properties, emotions can have a positive or a negative valence, depending on the value of $u$ and $u^{\prime}$. They also capture the idea that emotions have an 'intensity' (for psychologists, valence does not only capture the 'sign' of an emotion but can also be positive or negative at various degrees). Basic emotions as they are defined here are thus reminiscent of Kahneman and Tversky's view that individuals consider pleasure and pain not only in an absolute manner but also as 'gains' and 'losses' compared with the current situation (Kahneman \& Tversky 1984). For instance, the passage from a pleasant situation to an even more pleasant one will be greeted with joy, whereas the passage from a pleasant event to a less pleasant event will be experienced as frustration. However, if the passage is from a pleasant to a painful situation, the person will experience 
grief. On the other hand, if a person imagines the passage from a painful situation to a less painful or even pleasant situation, he will experience relief. The emotions described here are thus generated by a differential between two situations, but these situations do not have any specific reference to a particular time, date or duration. We call these emotions timeless basic emotions.

In the psychology literature, the term basic emotions has been used in various contributions, but it does not have a standard meaning (Ortony \& Turner 1990). For instance, basic (or primary) emotions relate to adaptative biological processes for Plutchik (1980), whereas for James (1884) they rely on bodily involvement (see Ortony \& Turner 1990, for other definitions). Although we share with other authors the identification of some of the basic emotions such as joy (Plutchik 1980) or grief (James 1884), in our context, emotions are considered basic emotions if they do not involve any temporal content.

\section{INTIMATE TIME, ANTICIPATION OF FUTURE EVENTS AND EMOTIONS}

What is, therefore, the relationship between time and basic emotion? Here, in fact, when we talk about time, we actually mean anticipated duration. The anticipated duration is the intimate interval of time the agent 'affectively' experiences between a current situation that generates $u$ at date $t$ and an imagined situation that will generate $u^{\prime}$ at a future date $t^{\prime}$. This concept is reminiscent of what psychologists call 'waiting time periods' as explained in $\S 2$.

In our theory, this anticipated duration depends on two factors. One is the experience of physical time, which we call clock-time $T$ hereafter. Between dates $t$ and $t^{\prime}$, there is an interval of time $T=t^{\prime}-t$ on which the anticipated duration will depend. The second factor is the basic emotion induced by the variation of utilities $u$ and $u^{\prime}$ (we refer to the anticipated duration as $\Delta\left(T, e\left(u, u^{\prime}\right)\right)$ in what follows). What do we now mean by duration as an affective experience? By this, we mean the interplay between basic emotions $e\left(u, u^{\prime}\right)$ and the sentiment of the passage of (clock) time $T$. What differentiates the sentiment of the passage of time from (basic) emotions? Whereas an emotion refers to the pain or pleasure differential (variation), a sentiment is, according to Livet (2002), an emotion that has become 'stabilized', that is, the experience of the emotion has become autonomous from the original pain and pleasure differential. In that sense, the sentiment of the passage of time is an invariant.

It has been argued that variations (emotions) are essential to uncover the invariant, i.e. the sentiment. Varela (1999) in his phenomenological study of nowness evokes a 'shift of transparency' that makes time 'visible'. The carpenter hammering instinctively does not experience time. Only when he hits his fingershift of transparency - does he notice time. This relates to the old philosophical debate on time and change. If the world completely freezes, does time pass (Scott 1995)? That is, the question is whether we need to observe changes in order to say that time has passed. Translated into our context, the question is whether we need to experience emotions in order to feel that time passes? At first yes, because as we explained above, we need emotions to create a sentiment of time, i.e. we need the variance in order to create the invariant. However, suppose the individual is thereafter held in a vacuum. We might suppose that a form of hysteresis of the sentiment of time initially persists such that the individual notices the passage of time. But when hysteresis has ebbed away, the individual will literally be left in a timeless vaccum. However, living individuals are not in a vacuum, but live their lives in a changing world. Hence, even if they themselves did not have any emotions, there are external changes affecting the world (if only the ticking of a nuclear clock), which create the sentiment of the passage of time. Formally, this means that $\Delta(T, 0) \neq 0$, $\forall T>0$.

The experience of clock-time $T$, hence the sentiment of the passage of time, on which the anticipated duration depends, refers to the anticipated actual experience of the (clock) time interval between two different dates $t$ and $t^{\prime}$. When the clock-time interval increases, the anticipated duration increases and vice versa.

The relationship between basic emotions and anticipated duration is slightly more complicated. It helps us to link anticipated duration to particular emotional (time) experiences. If the anticipated situation represents an improvement in relation to the current situation, thus generating joy or relief, the person's sense of anticipated duration will increase. This means more precisely that an anticipated joyful experience will expand the experience of time and this will induce what we call impatience (formally, $\Delta(T, e)$ increases). A person will therefore experience impatience only when she imagines an improvement relative to her current situation that is to take place at a particular date. Note that our concept of impatience is notably different from the one usually employed in the economic literature (see Loewenstein 1992 for a review). In the standard discounted utility theory, impatience is solely the preference for the present: one simply cannot resist the desire for present gratifications. Impatience therefore seems to be like a general psychological characteristic of human beings or a personality trait that can vary in degree depending on individuals (Böhm-Bawerk 1970 [1889]; Fisher 1930).

On the other hand, if the anticipated situation represents a deterioration of the current situation, thus creating frustration or grief, the person's sense of duration will decrease. Thus, an anticipated painful or less pleasant event will shorten the time experience of the individual and this will induce what we call anxiety (formally, $\Delta(T, e)$ decreases). Intuitively, a negatively evaluated event with respect to the current situation seems to arrive too quickly and time thus feels shorter.

Hence, in our theory, impatience and anxiety can be seen as two meta-emotions generated by the combination of time with basic emotions, i.e. basic emotions trigger impatience and anxiety when time is added as an extra dimension. ${ }^{13}$ In that sense, an individual can be seen to be impatient for forthcoming joy 
and anxious about future grief or frustration. The definitions of these two meta-emotions imply a general property of the effect of basic emotions on anticipated duration. That is, the anticipated duration increases with the basic emotion: the more joyful the event, the longer the anticipated duration and vice versa. We thus assume that there is a positive correlation between intimate time and what the psychophysiologists call emotional valence, which is in accordance with the literature we reviewed earlier. Note also that the concern for (in)sensitivity to rewards and punishments emphasized by brain studies appears in our theory through the basic emotions. The change in situation seen as improvement or deterioration induces positive (joy, relief) or negative emotions (frustration, grief) that will generate an increase (impatience) or decrease (anxiety) of anticipated duration. In addition to this, it can also be assumed that as time goes by, the effects of basic emotions on duration are less salient. In other words, the extension effect of time induced by positive emotions is stronger in the short than in the long run (and respectively so for negative emotions). This would mean that we have a decreasing marginal effect of basic emotion on duration. We explore in the next section the behavioural consequences of our theory. To do so, we focus on a particular setting: deciding now how a person should schedule (two) future actions. This is particularly interesting insofar as standard discounting theory irrevocably predicts that the highest pleasures should come first.

\section{SEQUENCE OF ACTIONS AND 'TIME-REVERSAL'}

Suppose that you have to do a number of tasks and you have to decide in which order to do them. Some tasks are pleasant while others are unpleasant tasks or duties. As in the experiment by Loewenstein \& Prelec (1991), suppose for instance that you have to plan two visits to the city where you once lived on two successive weekends. You can either meet 'former work associates whom you like a lot' or 'an irritating, abrasive aunt who is a horrendous cook'. The question is how to spend these weekends.

More generally, consider a situation in which a person has to decide now when to undertake two actions $A$ and $A^{\prime}$. One has to be done at a date $t$ while the other has to be done at some date $t^{\prime}$ and the person has to decide now $(t=0)$ in which sequence she will undertake these two actions. The two actions can be located on the scale of pain and pleasure, respectively, by the instantaneous utilities $u$, with value $P$, and $u^{\prime}$ with value $D$. The utility levels $P$ and $D$ are such that $D<P$ but can either be positive or negative. Depending on the sign of $P$ and $D$, we will therefore be in the space of pleasures, in the space of pains, or both. Going back to Loewenstein \& Prelec's experiment, it is important to note that there are actually three situations to consider (and not two as it may first appear): the visit to the aunt, the visit to former work associates, but both are considered from the current situation in which the person is right now. If we take this current situation as the reference point with $u=0$, the visit to the aunt constitutes a pain $(D<0)$ and the visit to former work associates a pleasure $(P>0)$ in relation to the current situation in which the person experiences no pleasure or pain.

In the standard discounted utility (DU) framework, the decision is clear: the more pleasant task will be done first followed by the less pleasant one. In this framework, the intertemporal utility is:

$U\left(u, u^{\prime}\right)=B(t) u+B\left(t^{\prime}\right) u^{\prime}$

where $B$ is the discount factor that actualizes future utilities. In the standard framework, $B$ takes the form $B(t)=e^{-\tau t}$ where $\tau$ is the positive discount rate. An alternative definition of the discount factor (in discrete time) is $B=[1 /(1+\delta)]^{t}$. In both specifications, the discount factor $B$ decreases with the time horizon, which means that people attribute more weight to the near future than to the distant future. The key point is that the discount factor in both cases is an exponential transformation of time. ${ }^{14}$ This is important insofar as exponential discounting is known to be the only transformation that satisfies time-consistency axioms (Strotz 1956). Time-consistency following exponential discounting means that the relative weights attributed to two future dates $t$ and $t^{\prime}$ do not depend on the date $s \leq t$ at which this future is considered. This is obtained in particular with $B=e^{-\tau t}$, a case in which changes in the discount factor are constant with respect to the time horizon. ${ }^{15}$ It thus follows that the intertemporal utility of the sequence $(P, D)$, $U(P, D)=B(t)[P+B(T) D]$, with $T=t^{\prime}-t$, is always larger than the intertemporal utility of the sequence $(D, P), U(D, P)=B(t)[D+B(T) P]$. Hence, in the standard exponential discounting utility framework, we prefer to experience pleasures earlier, before smaller pleasures or pains.

However, this is precisely not what Loewenstein \& Prelec (1991) find in their experiment. When subjects in the experiment had to state their preference, 90 per cent chose to meet the aunt first and leave the pleasant meeting with friends for the next weekend. Such responses suggest a negative discount rate, that is, the discount factor is such that the distant future is more 'important' than the near future (formally, $B(T)>1$ ). Similarly, Loewenstein (1987) provides examples of experiments in which many subjects prefer to delay by a few days a positive experience ('a kiss from the movie star of your choice'), or to get rid as soon as possible of a painful experience ('a (non-lethal) $110 \mathrm{v}$ shock'). Loewenstein (1987) develops a model in which anticipation in itself may generate positive or negative present utility, and calls these phenomena savouring and dread, respectively.

The path we follow here is quite different. We try to explain behaviour through the actualization of future utilities. That is, in our theory, anticipation affects intertemporal utility by changing the discount factor (and not through some 'utility from anticipation'). ${ }^{16}$ The question therefore is how an action or event at $t^{\prime}$ is evaluated from the point of view of $t$ ? In our theory, when this action or event leads to a change in instantaneous utility level from $u$ to $u^{\prime}$, it generates the basic emotion $e=e\left(u, u^{\prime}\right)$. This emotion acts on 
the anticipated duration through a function $\Delta(T, e)-$ recall that $T=t^{\prime}-t$ is the clock-time duration between the two dates. Following the previous section, we assume that $\Delta(T, e)$ is increasing in both clock-time $T$ and emotion $e$. The present value of $u^{\prime}$ is now given by $B\left[\Delta\left(T, e\left(u, u^{\prime}\right)\right)\right] u^{\prime}$ where $B$ is the discount factor associated with the anticipated duration $\Delta$. In our approach, the discount factor becomes an exponential tranformation applied to the anticipated duration $\Delta(T, e)$ instead of the clock-time duration $T$ only as in the standard approach. This means that $B\left[\Delta\left(T, e\left(u, u^{\prime}\right)\right)\right]=\exp \left[-\tau \Delta\left(T, e\left(u, u^{\prime}\right)\right)\right]$, where $\tau$ is the positive instantaneous discount rate. We therefore assume that the discount factor $B$ decreases with $\Delta$. Earlier pleasures are still preferred to later ones but the terms sooner and later are now associated with $\Delta$ rather than $T$. More precisely, the exponential specification implies that the discount factor $B\left[\Delta\left(T, e\left(u, u^{\prime}\right)\right)\right]$ decreases with $T$ (this is the standard horizon effect mentioned above), and decreases with the basic emotion $e$ (a stronger positive emotion increases the anticipated duration. It thus has a similar effect to an increasing clock-time horizon $T$ ).

So far we have explained what the discount factor is. What we now have to do is to define time preference. Time preference is generally defined as the effect of clock time $T$ on the discount factor $B$. In the standard approach, time preference is captured directly in the positive discount rate $\tau$. Note however that in this standard approach, time preference can be used interchangeably with 'impatience', that is, time preference is not seen as separate from psychological characteristics such as impatience, as mentioned in the previous section. For instance, Becker \& Mulligan (1997) say that '[a] patient person [...] has [...] a low rate of time preference' (p. 731, italics added). In our framework, however, time preference will be a combination of two effects: the effect of clock-time $T$ on $\Delta(T, e)$, which depends on the psychological effects of impatience and anxiety, and the effect of $\Delta$ on $B$, which, in our specification is captured by the discount rate $\tau$. Hence, given that the psychological effects are captured by the effect of $T$ on $\Delta$, the second effect captured by $\tau$ cannot any longer refer to impatience, as in the standard approach. The question therefore is what effect the discount rate $\tau$ accounts for. One could for example legitimate this discount rate $\tau$ with the philosopher Derek Parfit's argument of connectedness between the person now and some future state (Parfit 1984). In this view, a person is weakly connected throughout time if this person considers herself to be a 'different' person in the future. In such cases, Parfit claims that the person 'can rationally care less about [her] further future' (Parfit 1984, p. 313). This would explain the existence of $\tau .{ }^{17}$

Now that we have explained the elementary concepts surrounding the discount factor $B(\cdot)$, which consists of exponentially discounting the anticipated duration instead of the clock-time only, we are going to explain the mechanics of our approach and apply it to particular sequences of actions. As we have said, in our framework, basic emotions affect anticipated duration. Given that we discount anticipated duration, basic emotions will also have an impact on the discount factor. Assume that an event generates a positive (timeless) basic emotion (joy or relief). When this 'positive event' is in the future, the anticipated duration until this event happens increases, that is, the event generates impatience. This impatience is added to the positive discount factor $\tau$ and together they thus form a positive rate of time preference, which means that the discount factor $B(\cdot)$ as such decreases. ${ }^{18}$ This means that an event that generates a positive emotion appears to be further away and hence receives less weight in the distant future than the same event in the near future. In the symmetric case of a negative basic emotion (grief or frustration), a person feels anxiety and the event will appear to be much closer. If this negative effect dominates $\tau$, the rate of time preference may be negative. This means that the discount factor $B(\cdot)$ increases and, consequently, the distant future becomes more important than the near future. We call this phenomenon time-reversal.

Back to our problem; choosing the sequence of actions $(P, D)$ generates the discounted stream: ${ }^{19}$

$U(P, D)=B(t, e(0, P))[P+B(T, e(P, D)) D]$,

whereas choosing the sequence $(D, P)$ generates:

$U(D, P)=B(t, e(0, D))[D+B(T, e(D, P)) P]$,

where the referent utility level at the current date $(t=0)$ is normalized at zero in both cases. Even in this very simple set-up, four emotions have a potential impact on the decision: the comparison of $P$ and $D$ with the reference point $0, e(0, P)$ and $e(0, D)$, and the two comparisons of $P$ with $D$ depending on their time ordering, $e(P, D)$ and $e(D, P)$.

Notice that if emotions do not affect anticipated duration $\Delta(t, e)=t$, the discount factor $B(\cdot)$ will also be independent of the emotion $e$ and we immediately have the result of the standard discounting framework, that is, there is always a preference for doing more enjoyable tasks earlier (as soon as $B(T) \leq 1$ ). This underlines the fact that in order to obtain time-reversal (and, consequently, negative time preference), emotions must have a strong impact on time discounting. It also seems reasonable to assume that $B(t, e(0, P)) P$ is larger than $B(t, e(0, D)) D$, that is, when only one future action is considered, an action with higher utility $P$ will be preferred to another one with lower utility $D$. Also, if the future date is remote, i.e. if $T$ is large, then $B(T, e)$ will be assumed to be small for any basic emotion e. Consequently, the intertemporal utility of the sequence $(P, D)$, $U(P, D)$ will be larger than that of the sequence $(D, P), U(D, P)$. Thus time-reversal can only occur if the future date is not too remote, which seems also to be the case for savouring (Loewenstein 1987).

To give an example of time-reversal, suppose that for a time duration $t$, that is, from the referent time to a first event, emotions do not play an important role. In that case, we would have both $B(t, e(0, P))$ and $B(t, e(0, D))$ close to the same value, say $B$. Consider next the passage from a first to a second event and suppose that the difference between instantaneous utility levels $D$ and $P$ is very large, so that emotions 
$e(D, P)$ and $e(P, D)$ are strong (in absolute value). If $B(T, e)$ responds strongly to changes in $e$, this implies that $B(T, e(P, D))$ may be very small, whereas $B(T, e(D, P))$ may be large, say close to 1 . In that case, $U(D, P)$ is close to $B \cdot D$ (future outcome $P$ is very much discounted), whereas $U(P, D)$ is close to $B \cdot(P+D)$ (future outcome $D$, worse than $P$, is considered 'as salient' as immediate outcome $P$ ). If $P$ is negative, then $B \cdot D$ is preferable to $B \cdot(D+P)$, which implies time-reversal. Anxiety is so strong that it makes $D$ in the remote future as salient as $D$ in the near future, whereas impatience is so strong that it reduces any $P$, except if it occurs today.

The previous example illustrates an interesting feature: we assume that $B(t, e)$ was not sensitive to changes in $e$, whereas $B(T, e)$ was very responsive to such changes. If the duration between first and second event, $T$, is larger than $t$, the duration between the referent date and the first event, $B(t, e)$ will not be sensitive to changes in $e$, whereas $B(T, e)$ will be very responsive only if emotions have a stronger impact on the discount factor in the remote future (formally, the cross derivative $B_{T e}$ is negative). This, however, may not be a reasonable assumption. ${ }^{20}$ On the other hand, if $t$ is larger than $T$, then we may have time reversal with $B_{T e}$ positive. In that case, we need the two basic emotions $e(P, D)$ and $e(D, P)$ to have a strong impact on $B(T, \cdot)$, whereas we wish to keep the impact of basic emotions $e(0, P)$ and $e(0, D)$ on $B(t, \cdot)$ low. Though we have no formal proof of this conjecture, it seems that this is possible only if the distance between $D$ and $P$ is larger than the distance between either $P$ or $D$ to 0 , i.e. if $D<0<P$ : timereversal may occur only if one outcome is positive and the other negative (see appendix A for a numerical example).

\section{CONCLUSION}

In this paper, we incorporate emotions into intertemporal decision-making. The main feature of our approach is that time is not absolute but can rather have a certain 'elasticity' for the person, which will depend on the kind of emotions she experiences. Emotions are considered here to be affective resonances of differentials between situations (Livet 2002). Timeless comparisons of two situations or events give rise to basic emotions (grief, frustration, relief or joy). When this event is in the future, the basic emotion will trigger the time-related meta-emotions of impatience and anxiety. More precisely, a positive emotion such as relief or joy associated with an event that will happen in the future induces impatience. Impatience, in our context, implies that the experience of time up to the forthcoming event expands. A negative emotion such as grief or frustration associated with an event that will happen in the future triggers anxiety. This will give the experience of time contraction. Time, therefore, is not exogeneously given to the individual and emotions, which link together events or situations, are a constitutive ingredient of time experience. How time is experienced will depend on how events and situations are sequenced in the future. To a certain extent, our approach thus shares some elements of Bergson's concept of ‘duration' (Bergson 2001 [1927]).

We then explore some behavioural consequences of our theory. We do so by studying a specific decision problem, namely how best to plan given (pleasant and unpleasant) actions. This problem is interesting insofar as experimental evidence suggests that unpleasant or less pleasant actions are more likely to be preferred earlier. Yet, the standard exponential discounting framework leads to the inverse preference, namely greater pleasures are preferred earlier. Our approach, however, shows that when anxiety and impatience are strong enough, a person can prefer unpleasant situations to be in the near future rather than in the remote future.

Thus, our contribution here has been to suggest an interesting approach to introducing emotions into the experience of time. Much work remains however to be done. In particular, we have not explored timeconsistency problems, currently widely discussed in economics. For instance, there are situations where a person decides that she is going to undertake actions in a particular order, but will later disregard her initial decision when some actions have already been performed. Further exploration of the role of emotions in decision-making will in no doubt continue to yield valuable insights.

\section{APPENDIX A}

\section{(a) A numerical example of negative time preference}

In this appendix, we provide a numerical example of situations in which time-reversal occurs even under the more reasonable assumption that $B_{T e}$ is positive. As previously, consider firstly the basic emotion generated by the two successive actions $A$ and $A^{\prime}$, which generate utility levels $D$ and $P$, respectively. For the sake of simplicity, we assumed in this numerical example that an emotion is simply the difference between the two utilities of each action such that

$e\left(A_{t}, A_{t+1}\right)=u\left(A_{t+1}\right)-u\left(A_{t}\right)$,

where $A_{t}$ and $A_{t+1}$ are the actions undertaken at date $t$ and date $t+1$, respectively. The anticipated duration function $\Delta(\cdot)$ that combines the sentiment of the passage of (clock) time $T$ and the basic emotion $e\left(A_{t}, A_{t+1}\right)$ is defined such that:

$$
\begin{aligned}
\Delta\left(T, e\left(A_{t+1}, A_{t}\right)\right)= & T+\frac{\gamma}{\tau}\left[u\left(A_{t+1}\right)-u\left(A_{t}\right)\right] \\
& -\frac{\beta}{\tau} T\left[u\left(A_{t+1}-u\left(A_{t}\right)\right] .\right.
\end{aligned}
$$

In keeping with our previous definitions, the anticipated duration increases with $T$, increases when $u\left(A_{t+1}\right)$ increases and decreases when $u\left(A_{t}\right)$ increases. Tranforming the anticipated duration by an exponential function gives the discount factor: $B=$ $\exp \left(-\tau T+\gamma\left[u\left(A_{t+1}\right)-u\left(A_{t}\right)\right]-\beta T\left[u\left(A_{t+1}-u\left(A_{t}\right)\right]\right)\right.$. The parameter $\gamma$ controls for the impact of basic emotion on the discount factor. Namely, when $\gamma$ is large, basic emotions generate more anxiety or impatience. The interaction term $\beta T\left[u\left(A_{t+1}-u\left(A_{t}\right)\right]\right.$ allows 
(a)
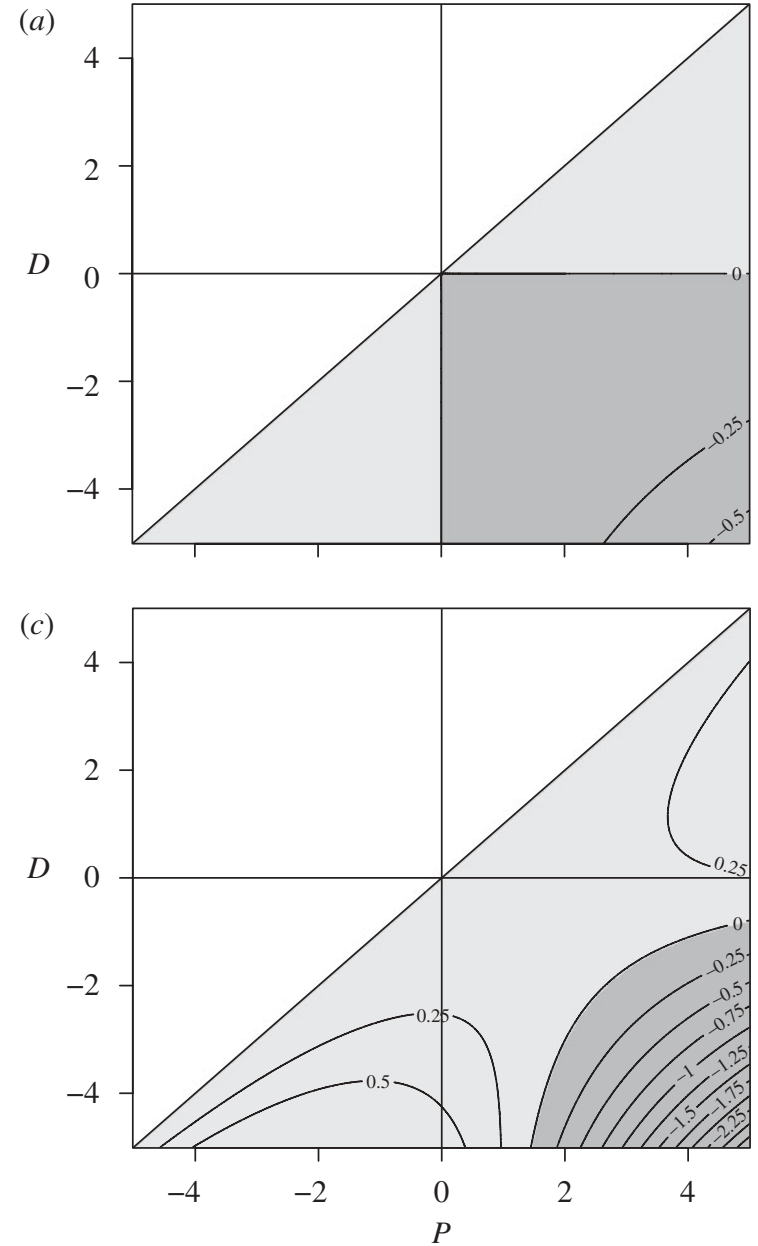

(b)

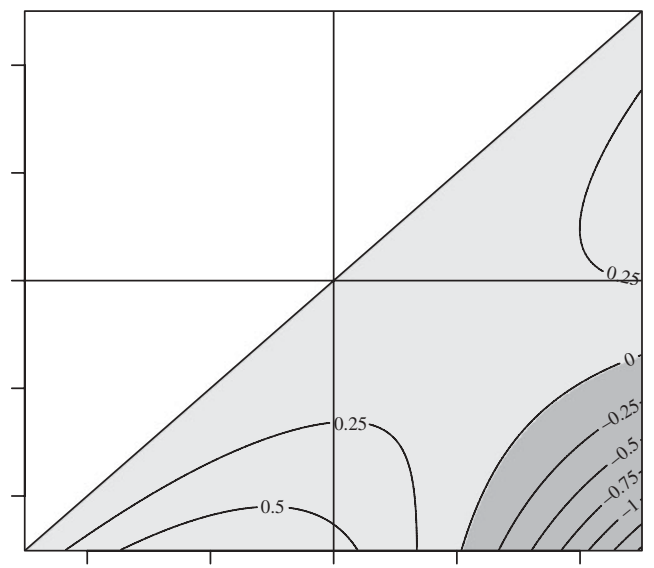

(d)

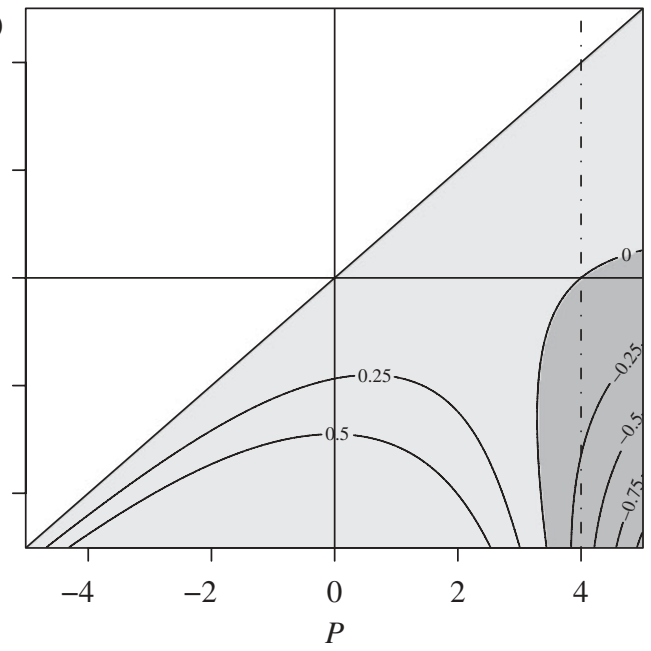

Figure 1. Time preference, emotions and sequence of outcomes. (a) Emotions only $(\gamma=0.04, \tau=\beta=0)$; (b) emotions and time preference $(\gamma=\tau=0.08, \beta=0)$; (c) emotions and time preference $(\gamma=0.1, \tau=0.08, \beta=0)$; (d) emotions and time preference $(\gamma=0.08, \tau=0.04, \beta=0.01)$.

us to illustrate the role of the decreasing impact of emotions on time when the time horizon increases.

Note that the duration function reduces to the standard discount factor when $\gamma$ and $\beta$ equal zero. Conversely, when $\tau$ and $\beta$ equals zero, only emotions matter. Each graph in figure 1 presents the difference $\Delta U=U(P, D)-U(D, P)$ for different sets of parameter values (with $t=0$ and $t+1=1$ ). The area in light grey (respectively, in deep grey) indicates that the utility difference is positive (respectively, negative), which means that the optimal sequence is to do $P$ first and $D$ second (respectively, to do $D$ first and $P$ second).

Figure $1 a$ only accounts for the impact of basic emotions on the anticipated duration, i.e the agent does not feel the passage of time. In this case, the standard result, that to do $P$ first, holds when $P$ and $D$ are both positive or negative in comparison with the initial level (denoted 0 in the figures). When $P$ is positive and $D$ negative, the standard result does not hold anymore and $D$ should be done first. Figure $1 b, c$ consider the case where both the sentiment of the passage of time and the basic emotions have an impact on anticipated duration. We do so for different values of $\tau$ and $\gamma$. They show that there exists an area, when $P$ is positive and $D$ negative, where the sequence $D P$ is still preferred over the sequence $P D$. Finally, figure $1 d$ illustrates the case where the cross-derivative $B_{T e}$ is not always negative (this is done by playing with the parameters in $B$ ). Note that the violation of the decreasing marginal effect of basic emotions when clock-time increases implies that the sequence $D P$ could also be chosen when both $P$ and $D$ are positive relative to the initial situation.

\section{ENDNOTES}

${ }^{1}$ Although the time production method leads usually to larger underestimations of the real duration than the analogue scale, these two methods are in general largely correlated (Osato et al. 1995). It seems also that the difference between the two methods varies with the complexity of the task (Sawyer et al. 1994).

${ }^{2}$ There is an International Affective Picture System (IAPS: Center for the Study of Emotion and Attention, CSEA-NIMH 1995) that has been standardized for self-assessed valence and arousal on large samples of subjects.

${ }^{3}$ In some studies, the heart rate is also used as a proxy of physiological arousal (see Fox \& Calkins 2000). This is however problematic insofar as heart rate changes are also a classic index of the amount of attention paid to a particular task (Angrilli et al. 1997).

${ }^{4}$ This is not so clear for the case of hyperbolic and quasi-hyperbolic discounting (see, Laibson 1997; Frederick et al. 2002, for a more detailed discussion of this). Usually, hyperbolic discounting is interpreted either as being a declining discount rate (hence fitting the second experimental setting), or as a 'momentary salience for 
the present' such as in Benabou \& Tirole (2002) (hence fitting the first experimental setting). However, it must be said that the fact that a person did stay longer at a party as in Ainslie's example (Ainslie 1992), contrary to his initial decision, might not have occurred if this same person had performed some other action, say running errands at the supermarket. In that case, the first type of experiments dealing with duration of stimuli may also be of interest for economists.

${ }^{5}$ The OFC is located within the frontal lobes, resting above the orbits of the eyes. It is defined as the part of the prefrontal cortex that receives projections from the magnocellular, medial, nucleus of the mediodorsal thalamus (see Rolls 2004, for a detailed description of the functions of the OFC).

${ }^{6}$ It is not our purpose here to insist on the 'efficiency' of emotions in decision-making. This is however a particularly interesting subject. Elster (1998) presents a stimulating discussion of this issue.

${ }^{7}$ In their study, there were mainly patients with dorsolateral frontal cortex (DLFC) lesions.

8Judgements on time therefore depend on 'pulse counting' (Varela 1999).

${ }^{9}$ However, some of their cognitive abilities are damagedfor example, non-OFC participants show an impairment in the spatial memory test. What this seems to indicate is that emotions and time perception are not related to the prefrontal cortex as a whole, but are situated particularly in the OFC (see Picton et al. 2006).

${ }^{10}$ See also Varela (1999) for a (neuro)phenomenological analysis of this argument.

${ }^{11}$ In that sense, emotions are not simply 'visceral factors' (Loewenstein 2000) such as hunger or drowsiness.

${ }^{12}$ Note that our analysis does however depart from the approach of Gilbert et al. (2002). They consider that 'our current reactions (to imagined events) are contaminated by our current (affective) circonstances' (p. 432) while, in our proposition, emotions are by definition a differential and hence are not some sort of bias.

${ }^{13}$ See Elster (1998) on the definition of meta-emotions.

${ }^{14}$ The exponential transformation implies that we can write $B(t)<$ $1=B(0)$ for a positive discount factor $\tau$ and $B\left(t^{\prime}\right)=B(t) B\left(t^{\prime}-t\right)$.

${ }^{15}$ Formally, $-\dot{B} / B=-(1 / B) \times \partial B / \partial T=\tau$ is constant. More general specifications that still satisfy time-consistency are recursive utility (Koopmans 1960) or variational utility (Geoffard 1996).

${ }^{16}$ Jevons (1905), quoted by Loewenstein (1987).

${ }^{17}$ This argument has spawned a lively debate in philosophy and we do not intend to dwell upon this issue too much in this paper. See Masson (1995) for an extensive review on time and identity, especially in the context of economics. Masson (1995) also provides other interesting arguments based on survival, existential nodes and life trajectories, which can justify certain types of time preference. However, it seems to us that the major difficulty that remains is where to draw the line between arguments concerning time preference as such and arguments concerning future 'utilities' (uncertainty, opportunity costs)—see Frederik et al. (2003).

${ }^{18}$ Remember that the discount factor is a decreasing function of anticipated duration.

${ }^{19}$ For the sake of simplicity, we now note $B[\Delta(t, e)]=B(t, e)$.

${ }^{20}$ Nevertheless, one could argue that when events are in the distant future, it may be cognitively difficult to represent them and to make explicit time-outcome trade-offs. Manzini \& Mariotti (2004) call this phenomenon time vagueness. In this situation, emotions may also have a strong impact as a heuristic for decision-making.

\section{REFERENCES}

Ainslie, G. 1992 Picoeconomics. Cambridge, MA: Cambridge University Press.

Angrilli, A., Cherubini, P., Pavese, A. \& Manfredini, S. 1997 The influence of affective factors on time perception. Percept. Psychophys. 59, 972-982.

Becker, G. \& Mulligan, C. 1997 The endogenous determination of time preference. Q. F. Econ. 112, 729-758. (doi:10.1162/003355397555334)
Benabou, R. \& Tirole, J. 2002 Self confidence and personal motivation. Q. F. Econ. 117, 871-915. (doi:10.1162/ 003355302760193913 )

Bergson, H. 2001 [1927] Essai sur les données immédiates de la conscience. Paris, France: Presses Universitaires de France.

Berlin, H., Rolls, E. \& Kischka, U. 2004 Impulsivity, time perception, emotion and reinforcement sensitivity in patients with orbitofrontal cortex lesions. Brain 127, 1108-1126. (doi:10.1093/brain/awh135)

Böhm-Bawerk, E. 1970 [1889] Capital and interest (South Holland ed.). IL, USA: Libertarian Press.

Curton, E. \& Lordahl, D. 1974 Effects of attentional focus and arousal on time estimation. F. Exp. Psychol. 103, 861-867. (doi:10.1037/h0037352)

Damasio, A. 1994 Descartes' error. New York, NY: Putnam.

Edmonds, E., Cahoon, D. \& Bridges, B. 1981 The estimation of time as a function of positive, neutral or negative expectancies. Bull. Psychonom. Soc. 17, 259-260.

Elster, J. 1998 Emotions and economic theory. F. Econ. Perspect. 36, 47-74.

Elster, J. \& Loewenstein, G. 1992 Utility from memory and anticipation. In Choice over time (eds G. Loewenstein \& J. Elster), ch. 1, pp. 3-34. New York, NY: The Russell Sage Foundation.

Feldman-Barrett, L., Batja, M., Ochsner, K. \& Gross, J. 2007 The experience of emotion. Annu. Rev. Psychol. 58, 373-403. (doi:10.1146/annurev.psych.58.110405. 085709)

Fisher, I. 1930 The theory of interest. New York, NY: Macmillan.

Fox, N. \& Calkins, S. 2000 Multiple-measure approaches to the study of infant emotion. In Handbook of emotions (eds M. Lewis \& J. M. Haviland-Jones), 2nd edn, pp. 203-219. New York: Guilford Press.

Fraisse, P. 1984 Perception and estimation of time. Annu. Rev. Psychol. 35, 1-36. (doi:10.1146/annurev.ps.35. 020184.000245)

Frederick, S., Loeweinstein, G. \& O'Donoghue, T. 2002 Time discounting and time preference: a critical review. 7. Econ. Literature 40, 351-401. (doi:10.1257/ 002205102320161311)

Frederik, S., Loewenstien, G. \& O'Donoghue, T. 2003 Time discounting and time preference: a critical review. In Time and decision: economic and psychological perspectives on intertemporal choice (eds D. R. George Loewenstein \& R. F. Baumeister), ch. 4, pp. 139-172. New York, NY: Russell Sage Foundation.

Frijda, N. 2000 The psychologists' point of view. In Handbook of emotions (eds M. Lewis \& J. M. Haviland-Jones), 2nd edn, pp. 59-74. New York: Guilford Press.

Geoffard, P. 1996 Discounting and optimizing: capital accumulation problems as variational minmax problems. F. Econ. Theory 69, 53-70. (doi:10.1006/jeth. 1996.0037)

Gilbert, D., Gill, M. \& Wilson, T. 2002 The future is now: temporal correction in affective forecasting. Organ. Behav. Human Decision Process. 88, 430-444. (doi:10. 1006/obhd.2001.2982)

Hornak, J., Bramam, J., Rolls, E., Morris, R., O’Doherty, J., Bullock, P. \& Polkey, C. 2003 Changes in emotion after circumscribed surgical lesions of the orbitofrontal and cingulate cortices. Brain 126, 1691-1712. (doi:10.1093/ brain/awg 168)

Hornak, J., O’Doherty, J., Bramham, J., Rolls, E., Morris, R., Bullock, P. \& Polkey, C. E. 2004 Reward-related reversal learning after surgical excisions in orbitofrontal and dorsolateral prefrontal cortex in humans. f. Cogn. Neurosci. 16, 463-478. (doi:10.1162/ 089892904322926791) 
James, W. 1884 What is an emotion? Mind 9, 188-205. (doi:10.1093/mind/os-IX.34.188)

Jevons, W. 1905 Essays on economics. London, UK: Macmillan.

Kahneman, D. \& Tversky, A. 1984 Choices, values and frames. Am. Psychol. 39, 341-350. (doi:10.1037/0003066X.39.4.341)

Koopmans, T. 1960 Stationary ordinal utility and impatience. Econometrica 28, 287-309. (doi:10.2307/1907722)

Laibson, D. 1997 Golden eggs and hyperbolic discounting. O. F. Econ. 112, 443-477. (doi:10.1162/ $003355397555253)$

Lang, P., Greenwald, M., Bradley, M. \& Hamm, A. 1993 Looking at pictures: affective, facial, visceral, and behavioral reactions. Psychophysiology 97, 261-273. (doi:10. 1111/j.1469-8986.1993.tb03352.x)

Langer, J., Wapner, S. \& Werner, H. 1961 The effect of danger upon the experience of time. Am. F. Psychol. 74, 94-97. (doi:10.2307/1419830)

Livet, P. 2002 Émotions et rationalité morale. Paris, France: Presses Universitaires de France.

Loewenstein, G. 1987 An inclusive theory of intertemporal choice should be able to account for both extremes of behaviour: myopic and far-sighted. Econ. F. 97, 666-684. (doi:10.2307/2232929)

Loewenstein, G. 1992 The fall and rise of psychological explanation in the economics of intertemporal choice. In Choice over time (eds G. Loewenstein \& J. Elster), ch. 1, pp. 3-34. New York, NY: The Russell Sage Foundation.

Loewenstein, G. 2000 Emotions in economic theory and economic behavior. Am. Econ. Rev. 90, 426-432. AEA papers and proceedings.

Loewenstein, G. \& Prelec, D. 1991 Negative time prefence. Am. Econ. Rev. 81, 347-352. AEA papers and proceedings.

Manuck, S., Flory, J., Muldoon, M. \& Ferrell, R. 2003 A neurobiology of intertemporal choice. In Time and decision: economic and psychological perspectives on intertemporal choice (eds G. Loewenstein \& R. F. Baumeister), ch. 4, pp. 139-172. New York, NY: Russell Sage Foundation.

Manzini, P. \& Mariotti, M. 2004 A theory of vague expected utility. Topics Theoret. Econ. 4. Available at: http://www. bepress.com/bejte/topics/vol4/iss1/art10.
Masson, A. 1995 Préférence temporelle discontinue, cycle et horizon de vie. In Le modèle et l'enquête (eds L.-A. GérardVaret \& J.-C. Passeron), ch. 9, pp. 325-400. Paris, France: Ecole des Hautes Etudes en Sciences Sociales.

O'Doherty, J., Kringelbach, M., Rolls, E., Horack, J. \& Andrews, C. 2001 Abstract reward and punishment representations in the human orbitofrontal cortex. Nat. Neurosci. 4, 95-102. (doi:10.1038/82959)

Ortony, A. \& Turner, T. J. 1990 What's basic about basic emotions? Psychol. Rev. 97, 315-331. (doi:10.1037/ 0033-295X.97.3.315)

Osato, E., Ogawa, N. \& Takoaka, N. 1995 Relations among heart rate, immediate memory and time estimation under two different instructions. Percept. Motor Skills 80, 831-842.

Parfit, D. 1984 Reasons and persons. Oxford, UK: Clarendon Press.

Picton, T., Stuss, D. T., Shallice, T., Alexander, M. P. \& Gillingham, S. 2006 Keeping in time: effects of focal frontal lesions. Neuropsychologia 44, 1195-1209. (doi:10.1016/j.neuropsychologia.2005.10.002)

Plutchik, R. 1980 A general psychoevolutionary theory of emotion. In Emotion: theory, research, and experience: theories of emotion (ed. R. P. H. Kellerman), vol. 1, pp. 3-33. New York, NY: Academic.

Reik, T. 1940 Aus Leiden Freuden. Masochismus und Gesellschaft. London, UK: Imago Pub. Co. 1td.

Rolls, E. T. 2004 The functions of the orbitofrontal cortex. Brain Cogn. 55, 11-29. (doi:10.1016/S0278-2626(03) 00277-X)

Samuelson, P. 1937 A note on measurement of utility. Rev. Econ. Stud. 4, 154-161.

Sawyer, T., Meyers, P. \& Huser, S. 1994 Contrasting task demands alter the perceived duration of brief time interval. Percept. Psychophys. 56, 649-657.

Scott, M. 1995 Time and change. The Phil. Quat. 45, 213-218. (doi:10.2307/2220420)

Strotz, R. 1956 Myopia and inconsistency in dynamic utility maximization. Rev. Econ. Stud. 23, 165-180. (doi:10. 2307/2295722)

Varela, F. 1999 The specious present: a neurophenomenology of time consciousness. In Naturalizing phenomenology: issues in contemporary phenomenology and cognitive science (eds B. P. J. Petitot, F. J. Varela \& J.-M. Roy), ch. 9, pp. 266-314. Stanford, CA: Stanford University Press. 